\title{
MODALPARTIKELN IM DAF-UNTERRICHT: VERMITTLUNG UND LEHRWERKANALYSE
}

\author{
Ebal Sant Anna Bolacio Filho \\ Thaís Lima \\ Belino dos Reis Barros
}

Resumo: $\mathrm{O}$ presente artigo trata das chamadas Modalpartikeln (MPn) da língua alemã e de seu ensino nas aulas de alemão como língua estrangeira (ALE/DaF). Após uma breve discussão sobre teorias que apresentam propostas distintas de explicação e ensino das MPn, passa-se à análise de uma obra didática atual a fim de se avaliar se e como as MPn são apresentadas na obra e os aportes teóricos das últimas décadas foram considerados na concepção do material didático.

Palavras-chave: alemão como língua estrangeira; ensino de línguas; linguística aplicada; formação de professores de alemão como língua estrangeira

Zusammenfassung: Der vorliegende Artikel beschäftigt sich mit den Modalpartikeln (MPn) der deutschen Sprache und derer Vermittlung im DaF-Unterricht. Nach einer kurzen Diskussion über Theorien, die verschiedene Ansätze der Erklärung und Vermittlung der MPn, wird ein aktuelles DaF-Lehrwerk analysiert. Das Ziel der Analyse ist es, herauszufinden, ob und inwieweit die Erkenntnisse der Modalpartikelforschung der letzten Jahrzehnte bei der Konzeption des Lehrwerks berücksichtigt wurden.

Schlüsselwörter: Deutsch als Fremdsprache; Fremdsprachenunterricht; angewandte Linguistik; Deutschlehrerausbildung

\section{Einleitung}

Die (Modal)Partikelforschung wurde bereits in den 60er Jahren von Krivonossow (1963) mit seiner Dissertation Die modalen Partikeln in der deutschen Gegenwartssprache und Weydt (1969) in seinem Buch Abtönungspartikel. Die deutschen Modalwörter und ihre französischen

${ }^{1}$ Prof. Adjunto UERJ - ebolacio@gmail.com 
52 | Ebal Sant Anna Bolacio Filho

Entsprechungen gegründet. Seitdem befassten sich sehr viele Forscher mit der Syntax, der Semantik und der Pragmatik dieser früher so vernachlässigten Wortart. Wie Pittner (2010) zur Modalpartikelforschung in der Germanistik anmerkt:

Modalpartikeln haben eine rasante Karriere gemacht von einer Geringschätzung als „Flickworte“, die wie „Läuse im Pelz einer Sprache“ herumwimmeln (...) hin zu einem in der germanistischen Linguistik anerkannten Status als vor allem für die mündliche Kommunikation wichtige Elemente, die Informationen über die Einstellung des Sprechers zur Äußerung und ihre Verankerung in der Kommunikationssituation geben. Im Zuge der pragmatischen Wende war ein regelrechter Partikel-Boom mit einer Flut an Veröffentlichungen zu verzeichnen, die bis heute kaum abgeebbt ist. (PITTNER 2010: 3)

Seit der kommunikativ-pragmatischen Wende in den 1970er Jahren gewinnen die Modalpartikeln (MPn), auch Abtönungspartikeln genannt, in der Forschung ebenfalls im Bereich DaF-Didaktik immer mehr an Bedeutung. Für den modernen, kommunikativen DaFUnterricht sind die Modalpartikeln (MPn) nämlich äußerst relevant, denn sie tragen dazu bei, die Sprache zu lernen und zu lehren, „wie sie eigentlich gebraucht wird“ (THURMAIR 2010: 3). In der Tat, wenn man sie in der gesprochenen Sprache weglässt, wo sie nach Müller hauptsächlich auftreten, „führt dies zu recht merkwürdiger, nämlich sehr ,hölzern“ wirkender Kommunikation" (MÜLLER 2014: 3).

Trotzdem gibt es eine deutliche Diskrepanz zwischen der tatsächlichen - nämlich eher bescheidenen - Rolle der MPn im DaFUnterricht und dem „Ausmaß, in dem sie im lebendigen, vor allem dialogisch sich realisierenden alltäglichen Gebrauch der Sprache vorkommen" (FERNER 2002: 3). Hinzu kommt noch, dass sie nach unserer Erfahrung meistens erst auf höheren Niveaus im Sprachunterricht behandelt werden, wenn sie nicht vollkommen ignoriert werden.

$\mathrm{Zu}$ dieser Einschätzung kamen auch die Teilnehmer des Aufbaustudiums Ensino de Alemão como Lingua Estrangeira an der Universidade do Estado do Rio de Janeiro im Jahr 2015, als sie in einem der Module des Aufbaustudiums, das in Kooperation mit der FriedrichSchiller-Universität Jena konzipiert wurde, mit dem Thema Modalpartikeln 
und deren Vermittlung im DaF-Unterricht konfrontiert wurden. Für viele war es das erste Mal, dass sie sich explizit und ausführlich mit dieser Thematik auseinadersetzten.

Das Thema der Abschlussarbeit von dem oben erwähnten Modul bestand darin, auf der Basis der Lektüre wissenschaftlicher Texte sowie der Diskussionen darüber ein aktuelles DaF-Lehrwerk zu analysieren, um herauszufinden, ob und inwiefern die Forschung über die MPn in die Lehrwerke Eingang fanden und wie die MPn im untersuchten Lehrwerk dargestellt und thematisiert werden. Schließlich sollte die Auswirkung der Diskussionen, die im Rahmen des Moduls Modalpartikeln im DaFUnterricht stattfanden, auf die Praxis der Autoren als Deutschlehrer kurz bewertet werden. Der vorliegende Artikel entstand aus den Diskussionen und Lektüren jenes Moduls.

\section{Modalpartikeln und DaF-Unterricht}

Der Einsatz von MPn im DaF-Unterricht stellt zweifelsohne eine große Herausforderung dar. Laut Muhr (1989) hängt das damit zusammen, dass ihre Bedeutung nicht immer eindeutig ist und sehr stark vom Kontext und auch von der Intonation abhängt - was typisch für die mündliche Sprache ist - und auch, dass sie manchmal verschiedenen Wortarten angehören, was oft bei den Lernenden und Lehrenden zu Verwirrung führt. Selbst unter Forschern besteht nämlich keine Einigkeit darüber, „wie viele und welche Wörter zu den MPn gezählt werden“ können (MÜLLER 2014: 1). Es ist also nicht verwunderlich, dass die meisten Lehrer überfordert sind, wenn sie die MPn erklären sollen, weil sie - selbst wenn sie Muttersprachler sind - die MPn in ihrer Ausbildung als DaF-Lehrer in der Regel nie behandelt haben.

In dem vorliegenden Artikel möchten wir zuerst zwei Ansätze zur Vermittlung von Modalpartikeln im DaF-Unterricht präsentieren und diskutieren. Um einen kleinen Einblick darein zu gewinnen, welche Rolle die MPn und ihre Forschung in den heutigen DaF-Lehrwerken spielen, wird anschließend der erste Band des Lehrwerks Menschen A1 in Bezug auf die Behandlung von MPn analysiert. Hierfür haben wir uns für den ersten Band entschieden, weil wir der Ansicht sind, dass die Modalpartikeln von Anfang an behandelt werden sollten, da sie in den alltäglichen Gesprächen vorkommen, die ja die ersten Strukturen sind, mit denen DaF-Lernende konfrontiert werden. 


\section{Vermittlung der MPn im DaF-Unterricht}

In ihrem im Jahr 2010 erschienenen Artikel Alternative Überlegungen zur Didaktik von Modalpartikeln beschäftigt sich Maria Thurmair mit der Vermittlung von Modalpartikeln im DaF-Unterricht. Laut Thurmair bereite dieses Thema bekanntlich viele Schwierigkeiten, denn die Bedeutung und Funktion von Modalpartikeln ließen sich nicht so einfach vermitteln, was der Autorin zufolge in vielen Publikationen, Lehrbüchern sowie in den MP-Didaktiken nachzulesen sei. Außerdem spielen sie immer noch eine geringere Rolle im DaF-Unterricht: Sie kommen zwar in Dialogen und Lesetexten vor, werden aber - wie bereits oben erwähnt - eher selten explizit thematisiert, besonders auf den niedrigeren Niveaus.

Thurmair (2010) präsentiert und diskutiert zwei Wege zur Didaktik von Modalpartikeln: Kognitive Vermittlung und Erwerb oder Vermittlung und Erwerb durch Imitation bzw. Automatisierung durch Chunks. In einer kognitiven Perspektive sollten die Bedeutung und Funktion der MPn didaktisiert werden. Laut Thurmair seien diese kognitiven Bedeutungserklärungen manchmal zu komplex für Lerner und Lehrer, weil die Bedeutungen der MPn nicht wirklich isoliert werden könnten. Kontext, Inhalt und Intonation müssten miteinbezogen werden, was in einer kognitiven Perspektive nicht einfach zu schaffen sei. Außerdem behauptet sie noch, dass es ab einem gewissen Grad der Komplexität Regeln und metasprachliche Erklärung nichts mehr zum Spracherwerb nutzen würden.

Thurmair stellt sich die Frage, ob die vorhandenen MPÜbungstypen "dem Erwerb einer MP-Kompetenz dienen” (THURMAIR 2010: 3), denn oft seien sie sehr anspruchsvoll und verlangten Sprachkenntnisse, die noch nicht erreicht worden seien. Das Problem bestehe ihrer Ansicht nach darin, dass bei diesen Übungstypen versucht wird, den Lernenden die Modalpartikeln kognitiv zu erklären. Dann würden die Bedeutungen jeder einzelnen Partikel ausführlich beschrieben, was zu Erklärungen führe, die den Spracherwerb nicht fördern würden. Dabei würde man auch sehr oft den Kontext und die Intonation vernachlässigen.

Als eine Alternative dazu, favorisiert sie die Vermittlung von MPn durch Wiederholung von Chunks. Dadurch könnte man eine kontextbezogene Beschreibung der Funktion jeder einzelnen Partikel bieten. Die Lernenden sollten dann formelhafte Strukturen mit MPn, die 
Modalpartikeln im DaF-Unterricht: Vermittlung und Lehrwerkanalyse | 55

rekurrente, strukturelle Muster darstellen, imitieren und dadurch automatisieren.

Chunks sind „Bündelungen von Informationen zu zusammenhängenden sprachlichen Strukturen" (LANG 2014: 4). Das heißt, anstatt zu versuchen, die Bedeutung und Funktion der Partikeln nur kognitiv zu erfassen, sollten die MPn durch Imitation und Wiederholung automatisiert werden. Dies würde geschehen, indem die Lernenden bestimmte Merksätze auswendig lernen, die ein prototypisches Beispiel für die Verwendung der Partikeln darstellen.

Obwohl dieses „Sätze-Merken-Verfahren“ heutzutage von vielen als ungeeignet und altmodisch gilt, betont Thurmair, dass die Merksätze sich dennoch produktiv verwenden lassen und in verschiedenen Kontexten modifiziert und erweitert werden können. Ein Beispiel dafür ist der Aussagesatz Männer sind eben so, der in einem Unterricht zum Thema Klischees und Vorurteile systematisch verändert werden kann: Männer/Frauen/Lehrerinnen/Italiener ... sind eben so/eitel/klug usw.

Dieser Ansatz (Chunk-Lernen) entspricht den heutigen Erkenntnissen darüber, wie Wortschatz erworben wird: sehr oft in „vorgefertigten“ formelhaften Strukturen. Dies zeigt folgende Zitate von Bohn (2000) und Apeltauer (2010) zum Thema Wortschatzlernen:

Betrachtet man den Wortschatz genauer, so wird deutlich, dass es nicht korrekt ist, nur von Wörtern zu sprechen, wenn damit auch Wortgruppen, idiomatische Wendungen (ein Brett vor dem Kopf haben) und Sätze (z. B. Sprichwörter) gemeint sind."

(BOHN 2000: 21)

Lernesprachen enthalten anfangs einzelne Wörter und Wendungen, die in wiederkehrenden (ritualisierten) Zusammenhängen gebraucht werden. Diese Wendungen (auch Formeln oder Chunks) gelten neben Inhaltswörtern als Grundbausteine des Zweitspracherwerbs, weil sie später aufgebrochen und variiert werden können und Lernern sowohl beim Erschliessen von Wortarten und Phrasenstrukturen helfen können. (APELTAUER: 838)

Auch die Modalpartikeln gehören unserer Meinung nach zu den Wortgruppen, die im Zusammenhang mit anderen Wörtern gelernt 
56 | Ebal Sant Anna Bolacio Filho

werden sollen, denn sie sind kontextabhängig und kommen meist in Strukturen vor, die eine sinnvolle Einheit bilden, d.h. in Chunks.

Wirft man einen Blick auf das vorhandene Lehrwerksangebot im DaF-Bereich, dann fällt sofort auf, dass Chunks schon einigermaßen bei der Wortschatzvermittlung verwendet werden. Meistens zeigen sie sich in den Lehrbüchern als Redemittel bzw. Ausdrücke, die so gelernt werden sollen, wie sie in bestimmten Situationen oft auftreten. Bei dem in diesem Artikel untersuchten Lehrwerk Menschen sowie bei anderen Lehrwerken werden sie z. B. in Redemittelkasten vorgegeben und am Ende jeder Lektion unter dem Begriff Kommunikation o. $\ddot{A}$ zusammengefasst. Bei der Frage Wie geht es dir? brauchen die Lernenden bekanntlich z. B. nicht von Anfang an zu wissen, dass dir die Dativform von $d u$ ist, was sie erst später erfahren werden. Das hat als Ziel, den Wortschatzerwerb und dadurch natürlich auch die Kommunikation in der Zielsprache zu erleichtern.

\section{Lehrwerkanalyse}

Das Lehrwerk Menschen wurde 2012 vom Hueber Verlag in Deutschland veröffentlicht. ${ }^{2}$ Es ist als Ausgabe in drei oder sechs Bänden erhältlich, die 24 bzw. 12 Lektionen beinhalten, und deckt die Niveaus A1 bis B1 nach dem Gemeinsamen europäischen Referenzrahmen ab. Es richtet sich an Erwachsene ohne Vorkenntnisse, die Deutsch als Fremdsprache sowohl in Deutschland als auch im Ausland lernen.

$\mathrm{Zu}$ diesem Lehrwerk gibt es viele Begleitmaterialien in gedruckter und digitaler Form, wie zum Beispiel Medienpakete, zweisprachige Glossare und differenzierte Arbeitsbücher für Lernende im In- und Ausland. Bei der Lehrwerkanalyse konzentrieren wir uns jedoch auf das Kurs- und Arbeitsbuch für Lernende im Ausland, auf die von Hueber angebotenen Unterrichtspläne sowie auf das Online-Angebot für Lernende.

Im Lehrwerk Menschen A1.1 werden die Modalpartikeln zuerst implizit eingeführt. Sie tauchen entweder in Texten oder in Dialogen vor, jedoch werden sie weder im Kursbuch noch im Arbeitsbuch explizit thematisiert. Es gibt überhaupt keine Erklärung für den Lernenden. Am Ende des Kursbuchs gibt es eine Wortliste, in der die MPn mit den

\footnotetext{
${ }^{2}$ Wir haben uns für die Analyse dieses Lehrwerks entschieden, weil es zurzeit an den meisten Sprachkursen (Sprachschulen, Sprachkurse für alle Fachbereiche an den Universitäten) in Rio de Janeiro als kurstragendes Lehrbuch verwendet wird.
} 
jeweiligen Seiten, wo sie zu finden sind, sogar erwähnt werden, aber auch ohne jegliche Erklärung, zum Beispiel, „aber (Modalpartikel) 26; denn (Modalpartikel) 25 und doch (Modalpartikel) 37", so dass der Lernende nicht weiß, was er mit der Information „Modalpartikel“ anfangen soll, wenn der Lehrer die MPn nicht thematisiert.

\begin{tabular}{|c|c|c|c|c|}
\hline denken & 63 & ein-steigen & 57 & feminin \\
\hline denn (Modalpartikel) & 25 & der Eintritt, - e & 39 & das Fernsehen (Sg.) \\
\hline der & 16 & die Einweihungsparty, -s & 91 & fern'sehen \\
\hline der Designer, - & 26 & das Eis (Sg.) & 51 & fertig (sein) \\
\hline die Designer-Brille, $-n$ & 30 & elegant & 30 & das Fest, -e \\
\hline das Designer-Modell, -e & 30 & die Eltern (Pl.) & 17 & das Festival, -s \\
\hline die Designer-Tasche, -n & 84 & die E-Mail, -s & 32 & das Feuerzeug, $-e$ \\
\hline As Dessert, -s & 51 & Ende: am Ende & 12 & der Film, e \\
\hline deutsch & 9 & endlich & 53 & die Film-Station, -en \\
\hline
\end{tabular}

(EVANS; PUDE; SPECHT (Kursbuch) 2012 : 98)

Man kann davon ausgehen, dass die meisten Brasilianer Begriffe wie Verb, Adverb, Präposition usw. kennen bzw. zumindest schon mal gehört haben, weil sie im muttersprachlichen bzw. fremdsprachlichen Unterricht in der Schule oft verwendet werden. Der Begriff Modalpartikel hingegen ist für sie vollkommen neu und unverständlich.

In der Lektion 4 im Kursbuch des ersten Bandes vom Lehrwerk Menschen tauchen die ersten Partikeln auf: denn und aber, die auch zu den häufigsten MPn gehören (Dudenredaktion 2009: 594). Diese kommen in einem Dialog zwischen Kundin und Verkäufer vor, der in einem Möbelhaus stattfindet. Durch den Gebrauch der MPn signalisiert die Kundin jeweils Interesse und Überraschung. Aber weder im Kurs- noch im Arbeitsbuch sind Übungen zu finden, bei denen diese Modalpartikeln explizit thematisiert werden. 


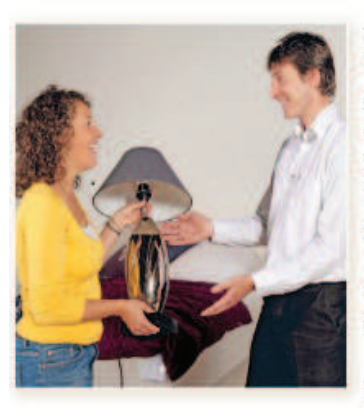

a __ Ja, bitte. Wie viel kostet denn der Tisch?

b 1 Brauchen Sie Hilfe?

c __ Der Tisch kostet 1478 Euro,

d __ Ja. Das ist zu teuer!

e __ Das ist aber sehr teuer!

$f$ Finden Sie?

$\mathrm{g}$ _ Sie kommt aus Italien. Der Designer heißt Enzo Carotti.

h _ Was kostet die Lampe?

i __ Die Lampe kostet nur 119 Euro. Das ist sehr günstig. Ein Sonderangebot. Die Lampe ist wirklich sehr schôn und nicht teuer!

(EVANS; PUDE; SPECHT (Kursbuch) 2012 : 26)

Der Unterrichtsplan der Lektion 4 enthält auf der Seite Nr.2 zwar einen kleinen Hinweis auf den Gebrauch der Partikeln in der deutschen Sprache sowie eine kurze Erklärung zu denn und aber; es wird aber keine Aktivität vorgeschlagen:

In der deutschen Sprache werden viele Partikeln benutzt. Sie modifizieren eine Aussage und zeigen eine subjektive Haltung: Denn in Fragen hebt das freundliche Interesse des Sprechers hervor (Wie viel kostet denn der Tisch?). Aber zeigt die Überraschung, das Erstaunen des Sprechers über etwas (Das ist aber sehr teuer.). Wirklich verstärkt sehr. Es ist noch kraftvoller als sehr, sehr schön (Die Lampe ist wirklich sehr schön.). ${ }^{3}$

Die Modalpartikel denn kommt wieder in der Lektion 6 vor, und zwar in einer Übung, bei der die Lernenden kleine Dialoge nach einem Muster spielen sollen. Auf den ersten Blick liegt der Fokus hier auf dem bestimmten Artikel im Akkusativ, aber die Partikeln denn und die neu eingeführte MP doch werden dabei in einer sehr lebensnahen Situation wiederholt, was offensichtlich beabsichtigt wurde.

3 Die Unterrichtspläne für alle Lektionen sind im Internet $z u$ finden: https://www.hueber.de/media/36/msn-unterrichtsplan-L4.pdf 


\section{Wo ist denn ...?}

a Hören Sie das Gespräch mit Frau Feser noch einmal und ergänzen Sie.

1 Wo ist denn

2 Sie haben Schlüssel? Schlüssel doch auch.

b Was suchen Sie? Spielen Sie ähnliche Dialoge. der Drucker I das Papier I der Kalender I die Rechnung

- Wo ist denn der Laptop?

$\Delta$ Der Laptop? Frau Esebeck hat doch den Laptop.

\begin{tabular}{|c|c|}
\hline Nominativ & Akkusativ \\
\hline $\begin{array}{l}\text { Da ist ... } \\
\text { - der Schlüssel } \\
\text { - das Papier } \\
\text { - die Rechnung }\end{array}$ & $\begin{array}{l}\text { Ich habe ... } \\
\text { - des Schlüssel } \\
\text { - das Papier } \\
\text { - die Rechnung }\end{array}$ \\
\hline $\begin{array}{l}\text { Da sind... } \\
\text { die Stifte }\end{array}$ & $\begin{array}{l}\text { Ich habe ... } \\
\text { die Stifte } \\
\text { auch so bei: brauchen, } \\
\text { suchen,... }\end{array}$ \\
\hline
\end{tabular}

(EVANS; PUDE; SPECHT (Kursbuch) 2012 : 35)

Während bei den Fragen die Partikel denn verwendet wird, wird die Antwort mit doch formuliert: Wo ist denn... (der Schlüssel/das Papier...)? / Sie haben... (den Schlüssel/das Papier...) doch auch. Die Modalpartikel denn kommt bekanntlich nur in Fragesätzen vor. Da es keine Hinweise im Unterrichtsplan der Lektion diesbezüglich gibt, muss der Lehrende hier selbst entscheiden, ob er diese wichtige Information mit den Lernenden teilt oder nicht, wenn die Kursteilnehmer nach deren Bedeutung fragen und falls der Lehrer sich selbst dessen bewusst ist.

Im Unterrichtsplan zur Lektion 6 wird den Lehrern empfohlen, die Kursteilnehmer die Gespräche in der Lektion 6 mehrmals in Partnerarbeit lesen und dabei möglichst genau die Intonation der Sprecher auf der CD nachmachen lassen. Dies hängt mit dem Ansatz zusammen, der oben präsentiert wurde, wonach die MPn eher implizit durch Wiederholung, Imitation und Automatisieren geübt werden sollen, sodass die KT eine Art „Sprachgefühl“ aufbauen können und sie dann „intuitiv“ benutzen.

In der Lektion 7 gibt es im Kursbuch eine Übung, bei der die Modalpartikeln tatsächlich im Mittelpunkt stehen, nämlich die MPn ja und aber. In der besagten Lektion 7 geht es um Freizeitaktivitäten und das Modalverb können. Bei der Übung 7 soll ein Lernender Hobbys vorspielen, während die anderen mit Komplimenten darauf reagieren.

Dies wird nach einem Beispieldialog gemacht und es gibt auch einen Hinweis auf die Stellung der Modalpartikeln im Satz. Im entsprechenden Unterrichtsplan gibt es eine Erklärung für Lehrende, wie Modalpartikeln in Kombination mit Adjektiven wie super und toll verwendet werden können, und auch den Hinweis, auf die Intonation der Lernenden zu achten. Sonst gibt es im Arbeitsbuch keine weiteren Übungen, bei denen die in der Lektion 7 eingeführten Partikeln eingeübt werden können. Mithilfe der oben genannten Hinweise im 
60 | Ebal Sant Anna Bolacio Filho

Lehrerhandbuch soll der Lehrer in die Lage versetzt werden, die Bedeutung und Funktion der MPn zu erklären.

7 Komplimente machen

Arbeiten Sie zu viert. Spielen Sie ein Hobby vor.

Die anderen machen Komplimente. Bedanken Sie sich dann.

- Du kannst ja toll singen!

- Du kannst wirklich toll singen!

^Vielen Dank! / Oh, danke! / Danke sehr! / Herzlichen Dank.

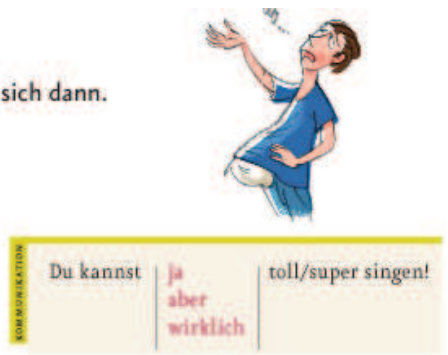

(EVANS; PUDE; SPECHT (Kursbuch) 2012 : 43)

In derselben Übung wird jedoch die Partikel wirklich eingeführt, die zwar eine Partikel (Adverb, Gradpartikel), aber keine Modalpartikel ist. Hier stellt man mal wieder fest, wie schwer es fällt, die Modalpartikeln von den anderen Partikeln auseinander zu halten (MORONI 2010: 3). Während wirklich als Adverb einen intensivierenden Charakter hat, drücken ja und aber eher die Überraschung/Erstaunen seitens des Sprechers aus. In diesem Kontext ist es jedoch nicht wichtig, dass die Kursteilnehmer eine ausführliche Erklärung bekommen, da die drei Partikeln als „pragmatisch fast gleichwertig“ vorgestellt werden. Es wäre aber schon wichtig, dass der Lehrende sich dessen im Klaren ist, dass es sich bei ja und aber einerseits und wirklich andererseits um unterschiedliche Partikeln handelt.

Im Arbeitsbuch ist auch zu sehen, dass es Übungen vorkommen, in denen die Modalpartikeln aber, denn und doch erscheinen. Jedoch eher selten, wenn es berücksichtigt wird, dass es dem Aufgabenrepertoire so viele Dialoge oder informelle Nachrichten gehören. Im ganzen Arbeitsbuch sind diesbezüglich nur fünf Übungen zu finden. Jede enthält einmal eine der erwähnten MPs. Wie im Kursbuch, werden sie auch nicht thematisiert oder systematisch geübt. Hier sind einige Beispiele: 
Modalpartikeln im DaF-Unterricht: Vermittlung und Lehrwerkanalyse | 61

\section{Ergänzen Sie den Chat.}

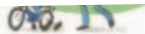

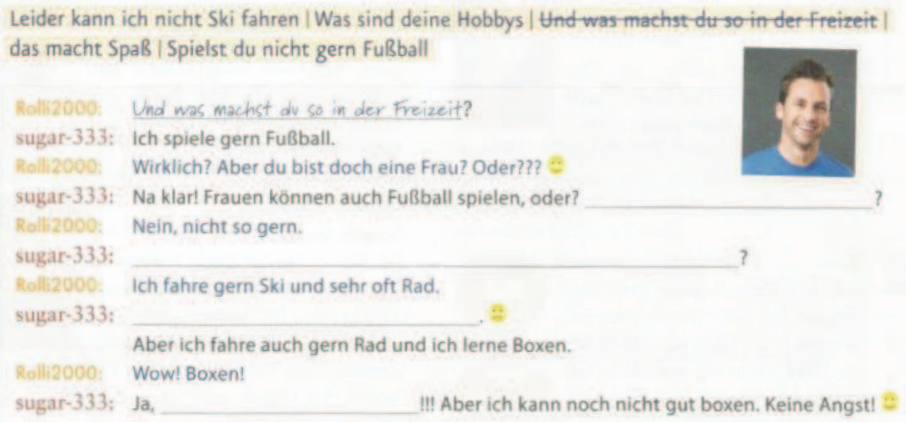

(EVANS; PUDE; SPECHT (Arbeitsbuch) 2012 : 55)

\section{TRAINING: SCHREIBEN}

\section{Einen Tagesablauf beschreiben}

a Lesen Sie Susanas E-Mail.
Liebe Christina,

wie geht es Dir denn in Deinem neuen Job als Au-pair-Mädchen in Köln? Was machst Du den ganzen Tag? Hast Du viel Arbeit? Viele Grüße Susana

b Christinas Tag. Ordnen Sie die Verben den Bildern zu.

im Supermarkt einkaufen | mit Freunden kochen I schlafen | zusammen essen | Frühstück-machen | Wohmung aufrăumen
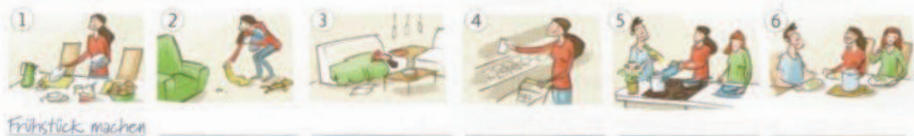

(EVANS; PUDE; SPECHT (Arbeitsbuch) 2012 : 87)

Hier ist uns wichtig hervorzuheben, dass es nicht immer Hinweise für Lehrer in den Unterrichtsplänen gibt, was MPn betrifft. Das kann zu Probleme führen, da die Lehrer nicht immer wissen, wie sie die Bedeutung bzw. Funktion einer Partikel erklären können und die MPn dann sehr oft vernachlässigen. 


\section{Schlusswort}

Die Analyse von Menschen A1 hat ergeben, dass die Modalpartikeln bei der Lehrwerkerstellung des untersuchten Lehrwerks didaktisch berücksichtigt wurden. Die Berücksichtigung der MPn beschränkt sich jedoch hauptsächlich auf das Kursbuch. Die MPn kommen im Arbeitsbuch als Übungen nicht vor und werden im Inhaltsverzeichnis auch nicht genannt. Die im Lehrerhandbuch vorhandenen Hinweise geben den Lehrenden zwar einige Informationen zur Bedeutung und teilweise zur Syntax der MPn, aber sie sind nicht ausreichend für eine erfolgreiche Vermittlung der MPn. Natürlich soll das Lehrbuch dem Lehrer nicht alles vorschreiben, aber es wäre sinnvoll, wenn die Unterrichtspläne mehr Informationen enthalten würden, um den Lehrenden bei der Vermittlung der MPn zu helfen.

Die Modalpartikeln werden im Lehrwerk Menschen teils implizit teils aber auch explizit präsentiert. Der Ansatz, der verfolgt wird, stützt sich offensichtlich auf die Vermittlung der MPn durch die Anwendung von Chunks: die Partikeln denn, ja, aber und doch - die bekanntlich zu den häufigsten MPn zählen - werden in Kontexten und Strukturen präsentiert, die sehr authentisch klingen. Auch wenn die vorgeschlagenen Aufgaben wie „Drills“ aussehen, sind sie unserer Ansicht nach nützlich und notwendig. Die Hinweise, die in den Unterrichtsplänen enthalten sind, stellen - wie oben bereits erwähnt - zwar eine Hilfe für die Lehrenden dar, sind aber nicht sehr ausführlich und schlagen keine weiterführenden Aufgaben vor.

Wir sind der Ansicht, dass eine Mischung aus Chunks und kognitiver Bewusstmachung die beste Lösung sein könnte, d.h. durch Beispiele, Kontextualisierung und sinnvolle Wiederholung kann man viele MPn vermitteln bzw. anschaulich machen. Es ist durchaus sinnvoll, die Modalpartikeln mithilfe von Chunks zu vermitteln. Sie erleichtern das Lernen und bieten die Möglichkeit, die Partikeln gleich zu Beginn einzusetzen, ohne die Lernenden mit zu vielen Informationen zu belasten. Auf dem Niveau A1 werden viele Strukturen wiederholt, ohne dass groß darüber nachgedacht wird. Dies gilt unserer Meinung nach für Strukturen wie „Wo ist denn der/die/das..?“, „Das ist aber schön/teuer usw.“ Es mag ja sein, dass kognitivere Lerner nach einer „logischen“ Erklärung fragen, da die Modalpartikeln sich in der Regel nicht eins zu eins ins Portugiesische übertragen lassen. Da muss der Lehrende eine Erklärung parat haben - was leider oft nicht der Fall ist. 
Modalpartikeln im DaF-Unterricht: Vermittlung und Lehrwerkanalyse | 63

Manchmal ist es unserer Meinung nach dennoch notwendig, die MPn kognitiv anzugehen, vor allem wenn man es mit erwachsenen Lernern zu tun hat. Ein gutes Beispiel hierfür findet man bei den Wunschsätzen: So oft werden sie mit den Partikeln doch und blo $\beta$ formuliert, dass sie in den Lehrbüchern so präsentiert und gelernt werden, ohne dass die isolierten Partikeln und ihre Funktionen erklärt werden. Oft reicht es einfach nicht aus, dass die Lernenden bestimmte Beispielsätze auswendig lernen. Bei Bedarf müssen die Lehrenden die Partikeln im Unterricht explizit thematisieren und dann üben lassen. In diesem Zusammenhang stellt LANG (2014) die Frage,

[...] ob ein korrektes Output des erwachsenen L2-Lerners als richtig angewendete und vor allem erworbene Regel gelten kann, oder ob es sich lediglich um die Reproduktion einer vorgefertigten Konstruktion handelt. Erwachsenen fehlt nämlich die Fähigkeit des Kindes, Chunks für eine Generalisierung und Regelbildung systematisch aufzubrechen und anzuwenden: Diese Feststellung muss also unbedingte Berücksichtigung finden, wenn Sprachwissenschaftler und Lehrende sich die Frage stellen, wie sie Fremdsprachlern die Sprache am besten und effektivsten beibringen. (LANG 2014: 4)

Und fügt gleich die Antwort hinzu:

So müssen auf jeden Fall Unterschiede gemacht werden in der Konzeption von einem Unterricht, der sich an Kinder wendet und solchem, der vornehmlich von erwachsenen Sprechern besucht wird.

In der Konsequenz müssen deshalb dem erwachsenen Zweitsprachlerner regulierend und lenkend solche vorgefertigten Konstruktionen vom Lehrenden zugeführt und den genauen Umgang mit diesen verdeutlicht werden (LANG 2014: 4).

Deswegen plädieren wir dafür, dass die DaF-Lehrwerke mehr Informationen über die MPn für die Dozenten enthalten und dass in der universitären DaF-Lehrerausbildung mehr Wert auf die Behandlung von Modalpartikeln (und überhaupt von Phänomenen der mündlichen Sprache) gelegt wird. 


\section{LITERATURVERZEICHNIS}

APELTAUER, E. Lernersprachen. In: KRUMM, H.-J. Deutsch als Fremdund Zweitsprache: ein internationales Handbuch 1. Halbband. Berlin: DeGruyter, 2010.

BOHN, R. Probleme der Wortschatzarbeit. München etc. (Fernstudieneinheit 22), 2000.

DUDENREDAKTION (Hg.) Duden: die Grammatik: unentbehrlich für richtiges Deutsch. [Der Duden in 12 Bänden. Das Standardwerk zur deutschen Sprache, Band 4]. Mannheim/Zürich: Dudenverlag, 2009.

EVANS, S.; PUDE, A.; SPECHT, F. Menschen A1. (Lehrbuch) Ismaning: Hueber, 2012.

EVANS, S.; PUDE, A.; SPECHT, F. Menschen A1. (Arbeitsbuch) Ismaning: Hueber, 2012.

FERNER, J. „Das hab ich doch gar nicht gelernt!“ Modalpartikeln und DaFUnterricht - Eine Problemskizze. 2002 URL: http://www.alfacert.unibo.it/moodle/pluginfile.php/85831/mod_res ource/content/2/fernermodalpartikeln1.pdf. Zuletzt überprüft am 27.11.2015.

LANG, F., Konsensus-konstituierende Modalpartikeln als prototypische Chunks: Eine Erwerbsperspektive für Deutsch als Fremdsprache. - Universität Freiburg, 2014. URL: https://portal.unifreiburg.de/sdd/fragl/lang2012.9. Zuletzt überprüft am 3.10.2016

LOCHTMANN, K./ DE BOE, S. Die soziolinguistische Kompetenz bei DaF-Lernenden und die Rolle der Modalpartikeln. In: Zeitschrift für Interkulturellen Fremdsprachenunterricht, Didaktik und Methodik im Bereich Deutsch als Fremdsprache, ISSN 1205-6545, Jahrgang 18, Nummer 2, Oktober 2013.

MORONI, M.C. Modalpartikeln zwischen Syntax, Prosodie und Informationsstruktur. Frankfurt am Main: Peter Lang, 2010

MUHR, R. (1989): Zur Didaktik der Modalpartikel im Unterricht Deutsch als Fremdsprache. In: WEYDT, H. (Hg.): Sprechen mit Partikeln. Berlin/New York: de Gruyter. S. 645-660

MÜLLER, S. (2014). Modalpartikeln. Heidelberg: Winter.

PITTNER, K. Modalpartikeln in neueren Lehrwerken für Deutsch als Fremdsprache. In: HINRICHS, N.; LIMBURG, A. (eds.): Gedankenstriche - Reflexionen über Sprache als Ressource. Für Wolfgang Boettcher zum 65. Geburtstag. Tübingen: Stauffenburg, 171-184. 
Modalpartikeln im DaF-Unterricht: Vermittlung und Lehrwerkanalyse | 65

THURMAIR, M. Alternative Überlegungen zur Didaktik von Modalpartikeln. Deutsch als Fremdsprache 47 (1), S. 3-9, 2010.

THURMAIR, M. Modalpartikeln und ihre Kombinationen. Max Niemeyer Verlag, Tübingen 1989.

WEYDT, H.;Harden, T.H.; HENSCHEL; RÖSLER, D. Kleine deutsche Partikellehre. Klett, Stuttgart 1983.

WEYDT, H. Abtönungspartikel. Die deutschen Modalwörter und ihre französischen Entsprechungen. Bad Homburg / Berlin West / Zürich 1969.

Recebido em: 12 de outubro de 2016.

Aceito em: 10 de dezembro de 2016. 